\title{
2018 - Un congrès de référence !
}

\section{S. Perrot (Rédacteur en Chef)}

(C) Lavoisier SAS 2018

Avec le congrès 2018, votre revue Douleur et Analgésie devient vraiment la revue de la SFETD. Un numéro spécial congrès SFETD 2018 qui permet de découvrir la diversité, la qualité et la créativité des travaux de la médecine de la douleur en France et dans le monde francophone.

Dans ce numéro, vous trouverez ainsi un résumé de la conférence inaugurale du Pr Herta Flor sur l'imagerie de la douleur, neuf résumés de séances plénières, dans des domaines très divers et enfin les 116 abstracts retenus par le comité scientifique.
Médecins, psychologues, infirmiers, kinésithérapeutes et chercheurs, tout le monde se retrouve dans ce numéro spécial qui confirme le dynamisme de la recherche clinique et fondamentale de la médecine de la douleur en France.

Votre revue avance, se développe, et avec ce numéro s'établit comme la référence incontournable, pour un congrès de référence.

Bonne lecture et bon congrès ! 